\title{
Excitation Energy Transfer Efficiency in Fluctuating Environments: Role of Quantum Coherence in the Presence of Memory Effects
}

\author{
Rajesh Dutta ${ }^{1}$ and Biman Bagchi ${ }^{1, *}$ \\ ${ }^{1} \mathrm{SSCU}$, Indian Institute of Science, Bangalore 560012, India. \\ Corresponding Author
}

*bbagchi@iisc.ac.in

SUPPORTING INFORMATION (SI)

Comparison between Rate and QSLE description

Survival Probability
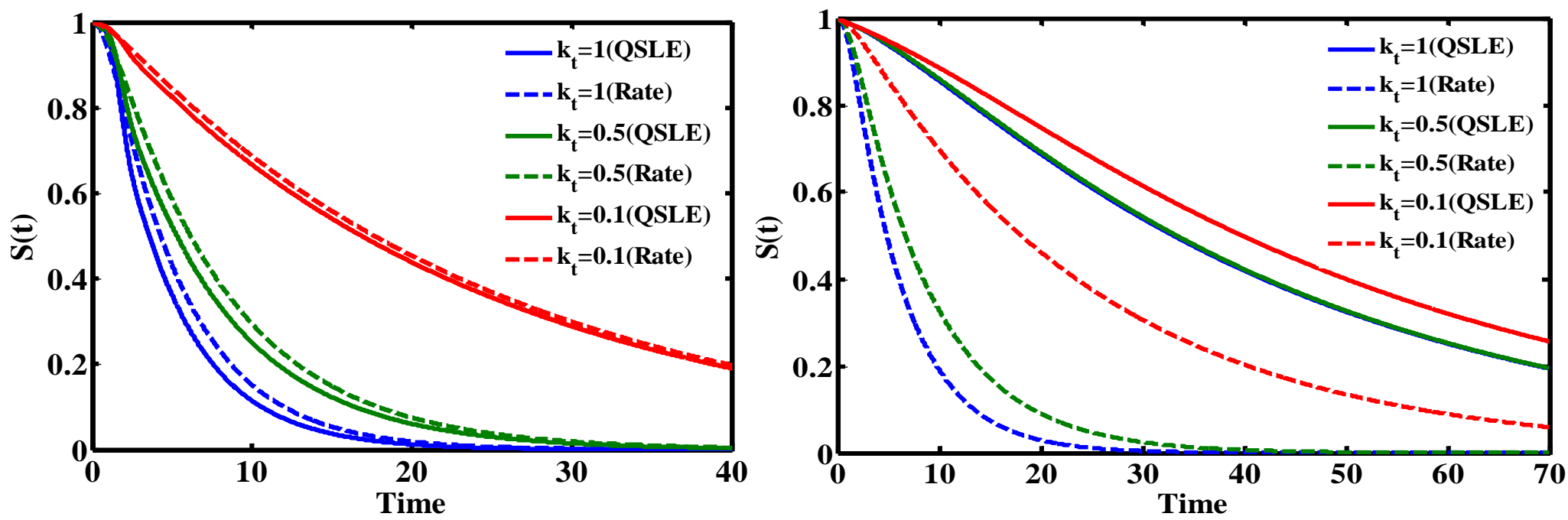

Figure-S1 Comparison of survival probability between QSLE and rate equation description for (a) strong coupling non-Markovian limit at $J=V_{d}=b_{d}=1$ (b) weak coupling Markovian limit $J=0.2, V_{d}=2$, $\mathbf{b}_{\mathrm{d}}=10$ for linear trimer with diagonal fluctuation at $\mathbf{k}_{\mathrm{d}}=\mathbf{0 . 0 1},\left|\Delta_{12}\right|=\left|\Delta_{23}\right|=1$ and $\left|\Delta_{13}\right|=0$. 

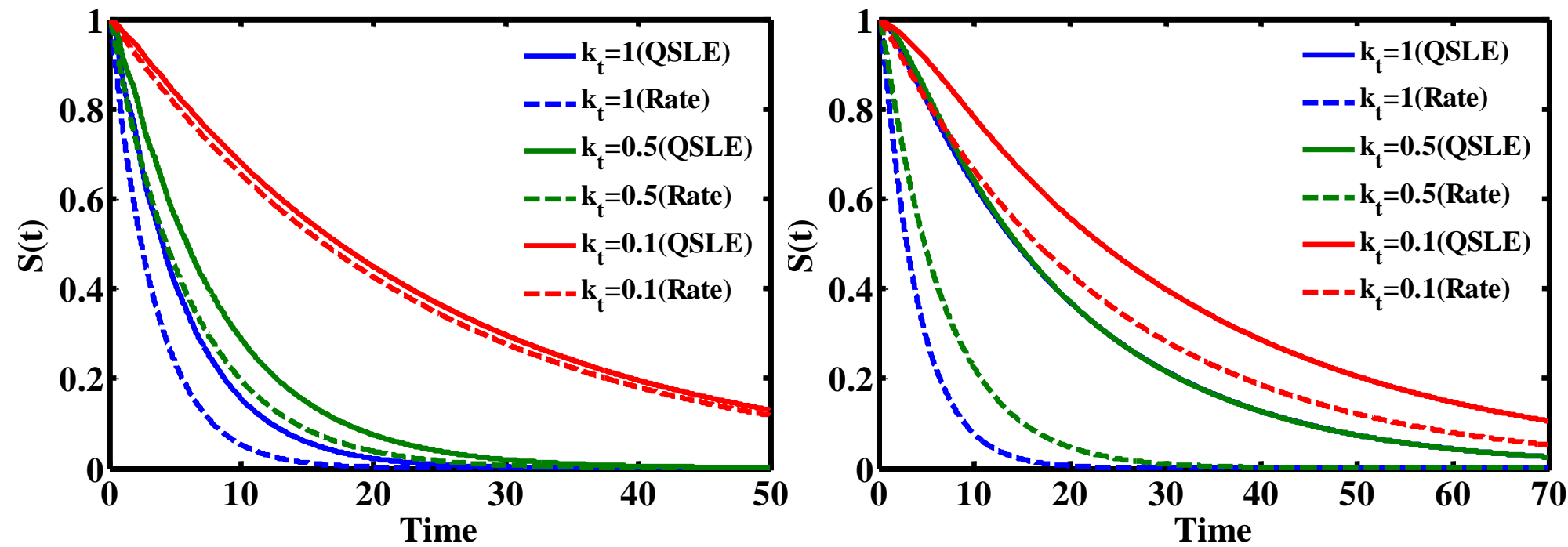

Figure-S2 Comparison of survival probability between QSLE and rate equation description for (a) strong coupling non-Markovian limit at $J=V_{d}=b_{d}=1(b)$ weak coupling Markovian limit $J=0.2, V_{d}=2$, $\mathbf{b}_{\mathrm{d}}=10$ for cyclic trimer with diagonal fluctuation at $\mathbf{k}_{\mathrm{d}}=\mathbf{0 . 0 1},\left|\Delta_{12}\right|=\left|\Delta_{23}\right|=1$ and $\left|\Delta_{13}\right|=0$.
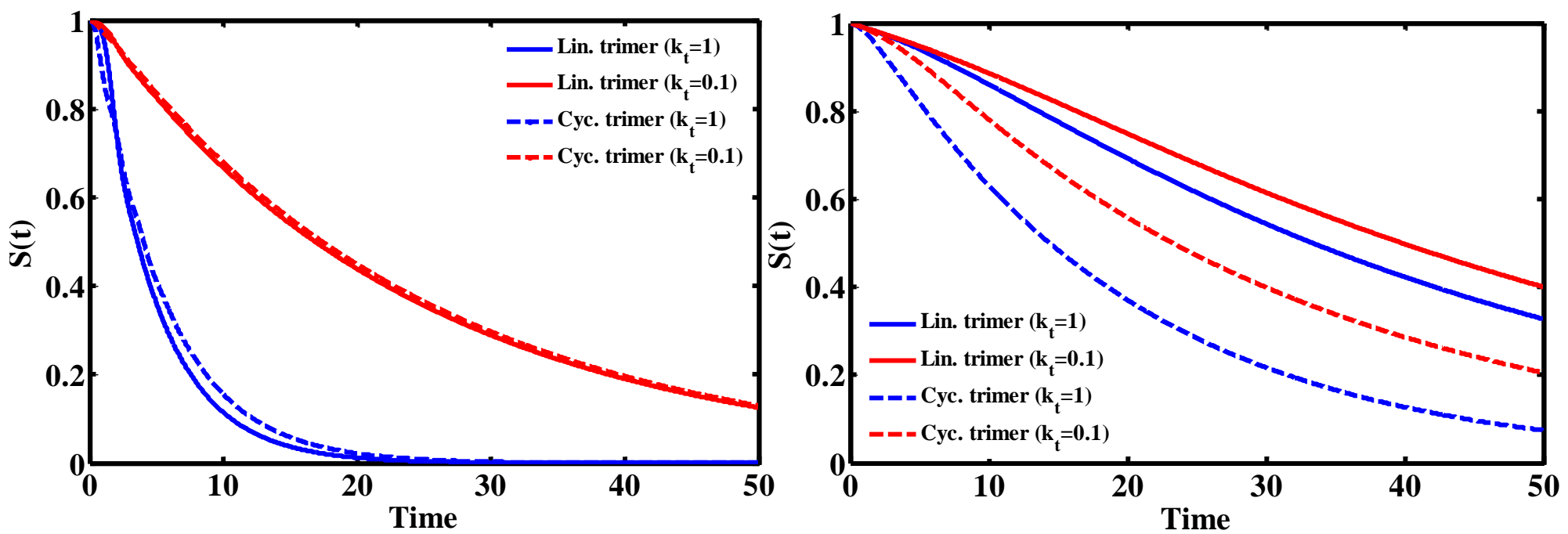

Figure-S3 Comparison of survival probability between linear and cyclic trimer for (a) strong coupling non-Markovian limit at $J=V_{d}=b_{d}=1$ (b) weak coupling Markovian limit $J=0.2, V_{d}=2, b_{d}=10$ with diagonal fluctuation at $\mathbf{k}_{\mathbf{d}}=\mathbf{0 . 0 1},\left|\Delta_{12}\right|=\left|\Delta_{23}\right|=1$ and $\left|\Delta_{13}\right|=0$. 


\section{Non-local coherences}
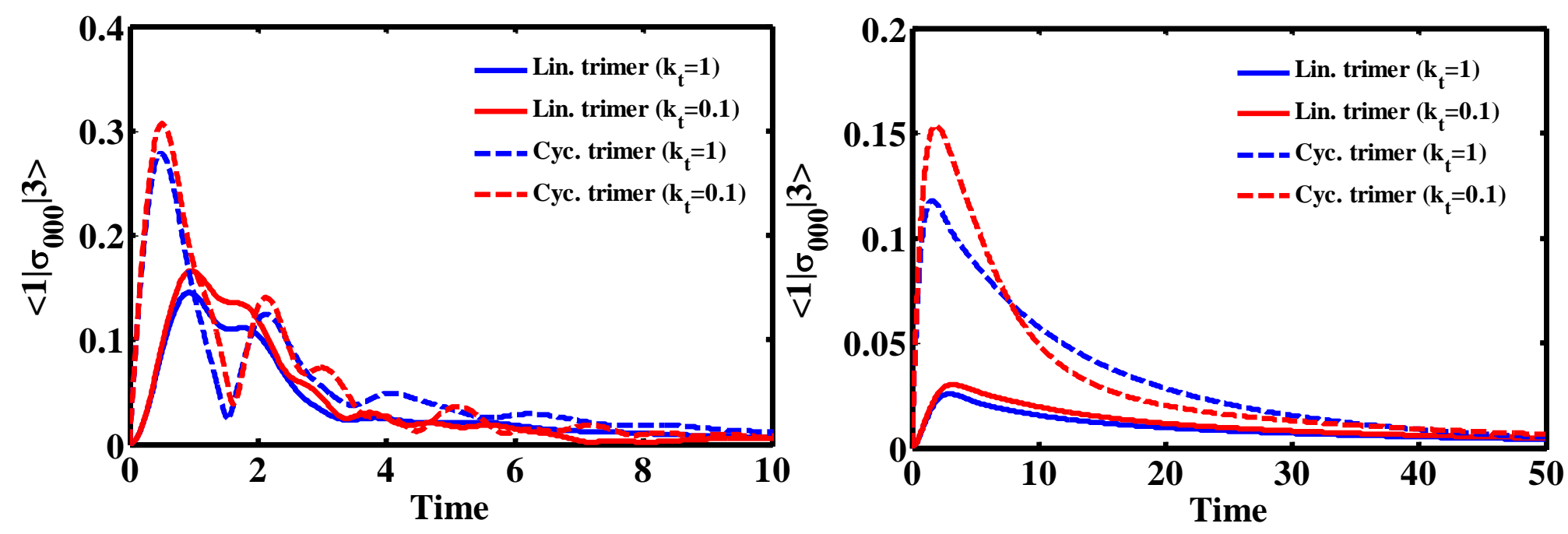

Figure-S4 Comparison of absolute value of non-local coherences in equilibrium bath states between linear and cyclic trimer for (a) strong coupling non-Markovian limit at $J=V_{d}=b_{d}=1$ (b) weak coupling Markovian limit $\mathbf{J}=\mathbf{0 . 2}, \mathrm{V}_{\mathrm{d}}=\mathbf{2}, \mathrm{b}_{\mathrm{d}}=10$ with diagonal fluctuation at $\mathbf{k}_{\mathrm{d}}=\mathbf{0 . 0 1},\left|\Delta_{12}\right|=\left|\Delta_{23}\right|=1$ and $\left|\Delta_{13}\right|=0$.

\section{Site-dependent population}

\section{Rate description (Dimer)}

$$
\begin{aligned}
& P_{1}(t)=\frac{k_{t}}{A} \exp \left[-\frac{B}{2}\right] \sinh \left(\frac{A}{2} t\right)+\exp \left[-\frac{B}{2}\right] \cosh \left(\frac{A}{2} t\right) \\
& P_{2}(t)=\frac{2 k_{1}}{A} \exp \left[-\frac{B}{2}\right] \sinh \left(\frac{A}{2} t\right)
\end{aligned}
$$

where,

$$
\begin{aligned}
& A=\sqrt{4 k_{1}^{2}+k_{t}^{2}} \\
& B=2 k_{1}+k_{t}+2 k_{d}
\end{aligned}
$$




\section{Dimer (off-diagonal fluctuation)}
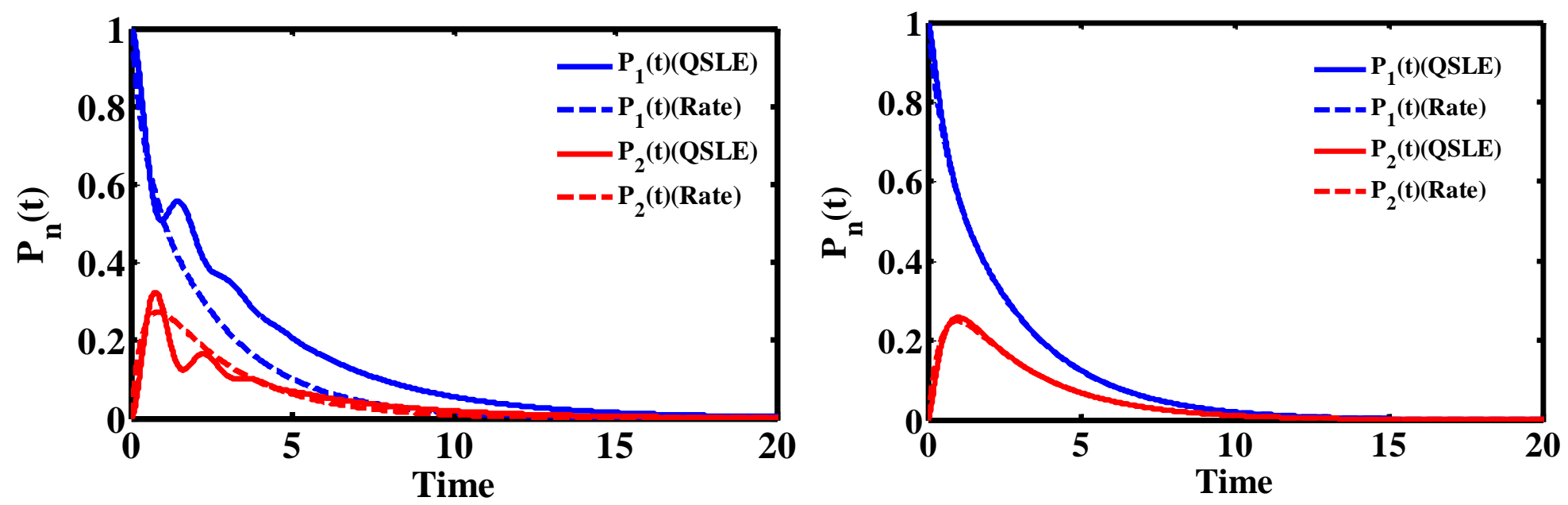

Figure-S5 Comparison of site wise population between QSLE and rate equation description for dimer with off-diagonal fluctuation (a) strong coupling non-Markovian limit at $J=V_{\text {od }}=b_{\text {od }}=\Delta=k_{t}=1$ and $\mathbf{k}_{\mathrm{d}}=\mathbf{0 . 0 1}$ (b) weak coupling Markovian limit for dimer with off-diagonal fluctuation at $\mathrm{J}=\mathbf{0 . 2}$, $V_{\text {od }}=2, b_{\text {od }}=10, \Delta=k_{t}=1$ and $k_{d}=0.01$. Solid line corresponds to QSLE description and dashed line corresponds to rate description. Initially site 1 was populated.

\section{Dimer (diagonal fluctuation)}
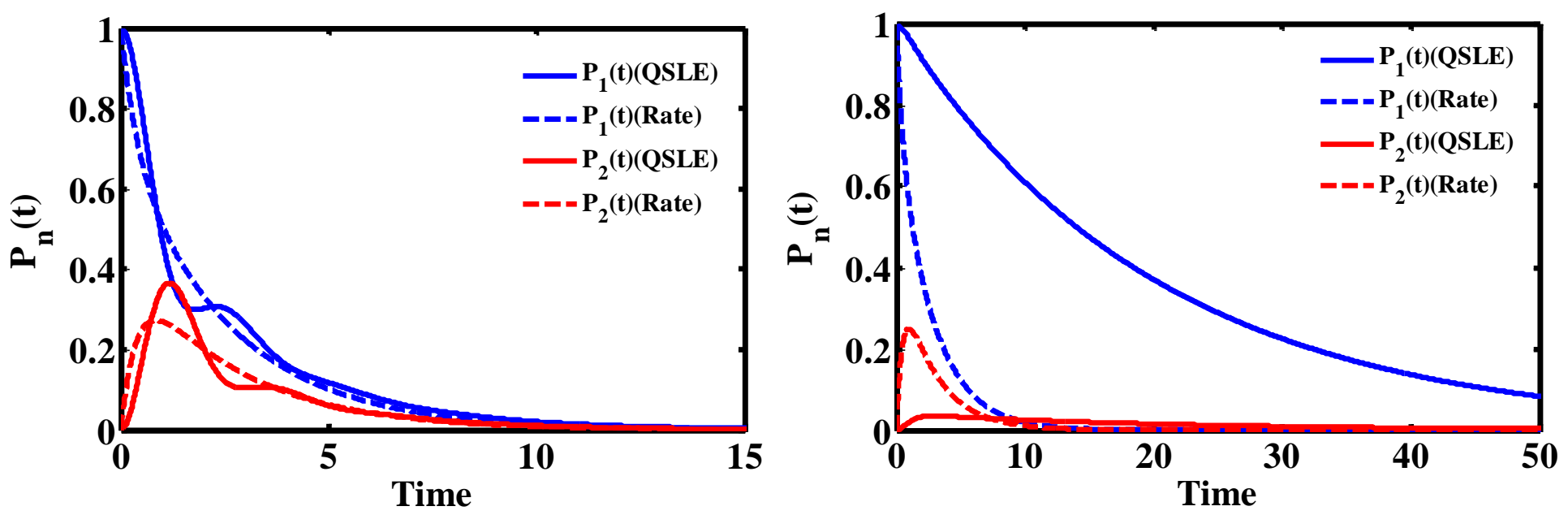

Figure-S6 Comparison of site wise population between QSLE and rate equation description for dimer with diagonal fluctuation (a) strong coupling non-Markovian limit at $J=V_{d}=b_{d}=\Delta=k_{t}=1$ and $k_{d}=0.01$ (b) weak coupling Markovian limit for dimer with diagonal fluctuation at $J=0.2, V_{d}=2$, $b_{d}=10, \Delta=k_{t}=1$ and $k_{d}=0.01$. Solid line corresponds to QSLE description and dashed line corresponds to rate description. Initially site 1 was populated. 


\section{Mean survival time}
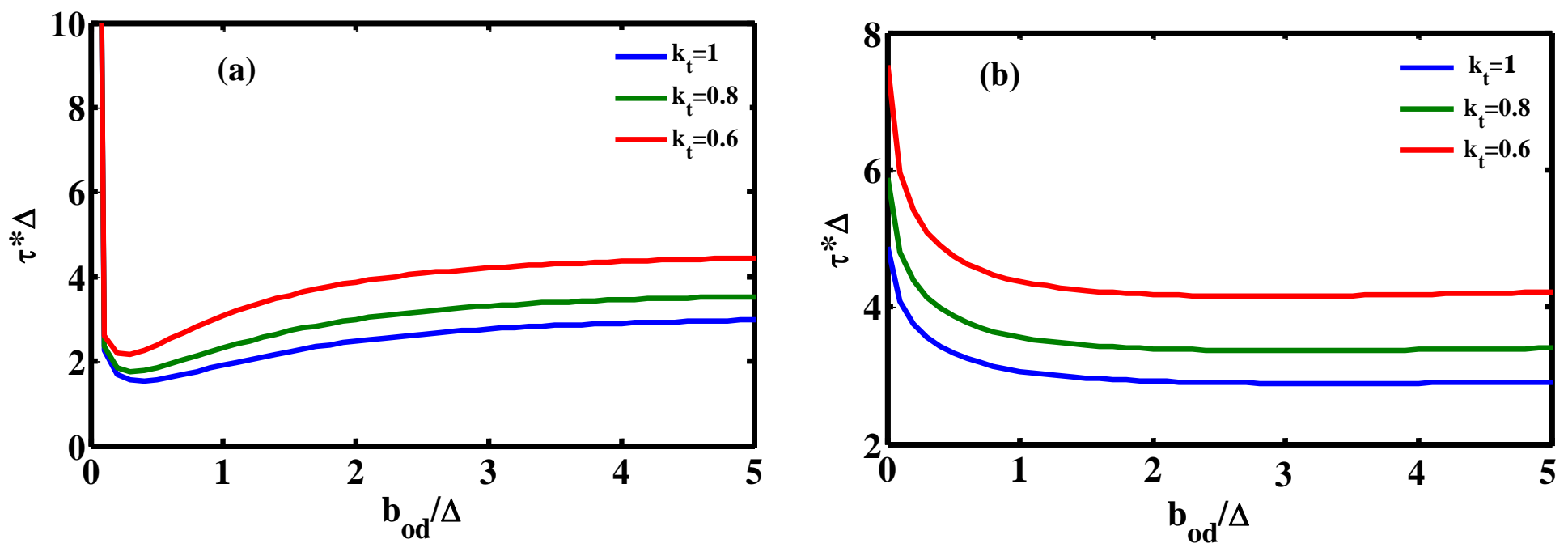

Figure S7. The mean survival time is plotted against inverse of bath correlation time at different trapping rate and decay rate $k_{d}=0.01$, in case of dimer with (a) off-diagonal fluctuation at $J=V_{o d}=1$ and (b) diagonal fluctuation at $J=V_{d}=1$. For figure (a), $y$ axis is considered upto 10 to show the difference between three trapping rates. 